\title{
Comparison of the Flow Inside Whirling and Non-Whirling Smooth Annular Seals at 50\% Eccentricity
}

GERALD L. MORRISON*, MARK C. JOHNSON, and H. DAVIS THAMES III

Texas A \& M University, Mechanical Engineering Department, College Station, Texas 77843-3123, USA

\begin{abstract}
The flow field inside whirling (whirl ratio $=1$ ) and nonwhirling seals can be dramatically different. The statically eccentric seal is dominated by viscous drag which is responsible for generating the azimuthal velocity inside the seal and the turbulence. Conversely, the whirling seal produces the azimuthal velocity component by pushing the fluid by the rotor (dynamic forces) which results in larger azimuthal velocity values, decreased radial velocity gradients, and decreased turbulence production inside the seal. The biggest difference is that the region of high pressure switches from one side of the seal to the other. This implies that at whirl ratios between 0 and 1 the magnitude of the pressure and suction pressures must decrease in value from the statically eccentric values, reach a minimum, and then increase again only on the opposite sides of the seal. This can explain why the value of the whirl ratio can determine if a seal will be stabilizing or destabilizing.
\end{abstract}

Keywords: Seal; Annular; Smooth; Fluid; Velocity; Whirl

In turbomachinery, smooth annular seals are used to decrease secondary leakage flow between high and low pressure regions. The geometry of a seal has several effects on the performance of rotating machinery. The most apparent is the slowing of leakage flow from the high to low pressure regions and the separation of flows that should not mix. Two other parameters that are affected by seal geometry are vibration and wear. Vibration can be

Received 5 July 2000; In final form 13 July 2000.

This research was supported by NASA Lewis Research Center, the Turbomachinery Research Consortium at Texas A \& M University, and the Exxon Education Foundation. These data and test section drawings are available on disk from Dr. Morrison.

*Corresponding author. Tel.: (409)845-5414, Fax: (409)845-3081. E-mail: gmorrison@mengr.tamu.edu caused when forces generated by the leakage are not uniform inside the seal and by non axisymmetric blade loadings that can be caused by tip leakage. The geometry also affects how the seal wears. If the flow through the seal causes stress at a particular point on the seal, cracks and breaks can occur, which will influence the performance of the seal. Seals with a small axial Reynolds number (generally below 2000) are generally unstable while seals with large axial Reynolds numbers produce large damping and stiffness coefficients and therefore contribute to stability (Allaire et al., 1978). Annular seals are also used as journal bearings in sealless pumps, which recirculate the lubricating fluid in the journals to provide cooling through the drive area.

Annular seals are common because of their simplicity (Figure 1). The rotor is usually the shaft of the machine, and the stator is usually the machine's housing. In sealless pump bearings, the rotor is a ring slipped onto the shaft and the stator is another ring mounted in the housing.

The mechanical performance of an annular seal is implicitly dependent on the fluid flow through the clearance, since the forces in the seal are generated hydrodynamically. Destabilizing forces tend to move the rotor of an annular seal from its centered position, and whirl usually follows. Whirl occurs when the center of the rotor precesses around the center of the stator. The whirl ratio is defined as the precession rate of the whirling rotor/shaft rotation rate. The rotor excitation can become so great that the rotor will contact the stator.

There is usually much effort devoted to characterizing and predicting the damping, stiffness and inertia variables in a seal to decide whether it will be stable prior to production. While the macroscopic effects have been studied extensively, the flow field within the seal has not been investigated as much because of the difficulty of the measurement. The clearances must be very small to simulate a real machine, and it is very difficult to measure velocities in the wake of the rotor. 


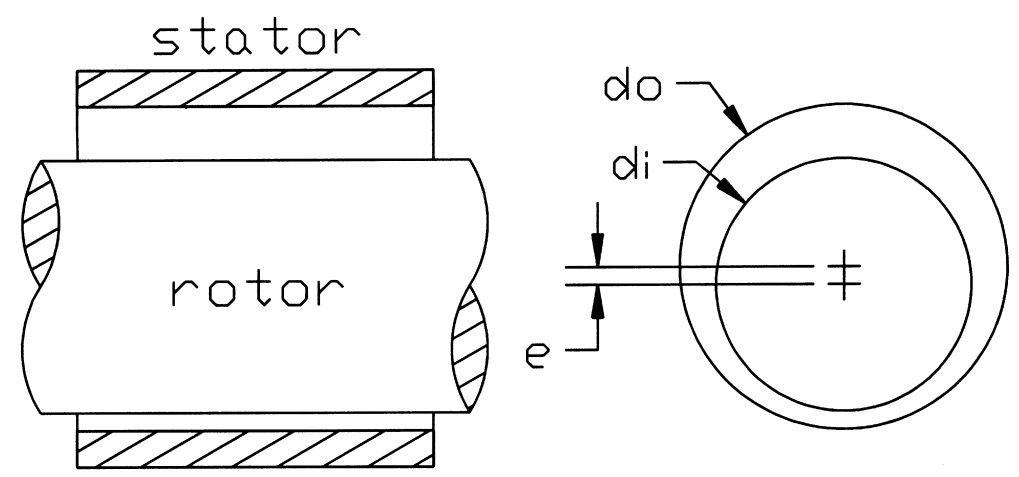

FIGURE 1 Schematic of a typical annular seal.

The present work investigates the effect of static and whirling eccentricity upon the flow field inside the seal by presenting measurements of the mean velocity and turbulence kinetic energy distributions between the stator and rotor of whirling and non-whirling plain annular seals operating at the same Reynolds and Taylor numbers. Radial averaged values of the axial and azimuthal mean velocities and the turbulence kinetic energy are presented along the length of the seal to demonstrate the effect of whirl upon "bulk averaged" values.

\section{PREVIOUS RESEARCH}

Annular seals have been investigated since the mid-1970s, when they were identified as contributors to turbomachine instabilities. In 1977 data became available for a wide range of operating conditions because of research directed toward the fuel pumps in the Space Shuttle's main engine (Tam, 1988). The following is a review of some relevant research regarding fluid flow through eccentric annular seals. The investigations mainly involved rotordynamic modeling.

Lessen (1987) performed an analytical study of the flow in a dynamically eccentric whirling annular seal at an arbitrary Taylor number within the Taylor vortex regime. Axial flow through the seal was modeled as plug flow which was justified by assuming high momentum transport from Taylor vortices. Boundary layers near the rotor and stator surface were dominated by Goertler disturbances (instabilities akin to Tollmein-Schlichting waves). Goertler disturbances were induced by the curvature of the boundary surface, and in this geometry are of far less importance than the Taylor vortices (Schlichting, 1979). The width of the Goertler disturbance-dominated regime was taken to be one unit of the non-dimensional Blasius variable, $y \sqrt{U / v x}$, at which point the local velocity $\mathbf{u} / \mathbf{U}$ was 0.33 . Lessen suggests that marginally unstable Goertler disturbances will be superseded by Tollmein-Schlichting waves if the sum of squared axial and tangential Reynolds numbers exceeds the critical Reynolds number for their formation:

$$
\mathrm{Re}^{2}+\mathrm{Re}_{\theta}^{2} \geq \mathrm{Re}_{\text {crit }}^{2}
$$

This indicates that the nature of the turbulence in the seal resembles flat plate turbulence at high tangential and axial Reynolds numbers. It was further found that the reaction forces in the radial direction were inertial in nature and could induce instabilities, and that the tangential reaction force was purely a function of viscous forces. Finally the ratio of potential core circulation to shaft circulation was the same in the eccentric rotor case as in the concentric.

Lessen's flow assumptions require that a very small axial Reynolds number be used. Axial flow through the clearance was modeled as Couette flow, defined as flow between surfaces in the absence of a pressure gradient. This is generally not the case in annular seals, since their primary function is to prevent leakage from high pressure areas in machines. Taylor vortices are also a transitionary phenomena between laminar and fully turbulent flow. Lessen used these vortices to justify a uniform tangential velocity profile over much of the clearance of the seal, but the laminar Taylor vortex regime is not very large. Schlichting (1979) quotes Stuart (1958) in setting limits on the laminar Taylor vortex dominated regime to be $41.3 \leq \mathrm{Ta} \leq 400$, an order of magnitude less than those characteristic of modern applications. Lessen's important finding was that tangential flow circulation did not change when the rotor was offset eccentrically (whirl was not considered).

Chen and Jackson (1984) studied the effect of axial eccentricity (displacement in the $r-\theta$ plane) and misalignment (angular displacement in the $x-r$ plane) on rotordynamic coefficients in annular seals. They suggested that the forces generated in high pressure seals are greatest in straight annular seals, and developed relationships between reaction forces and leakage rate through the seals that included the effects of eccentricity, misalignment and 
rotation. A concentric tapered annular seal was used as a model for the eccentric seal, and it was found that the effect of eccentricity or misalignment on the leakage rate was not as great when the flow regime was turbulent. The seal leakage increased with the degree of eccentricity, and decreased with the degree of misalignment.

Allaire et al. (1978) obtained an analytical solution of the semi-empirical bulk flow equations developed by Hirs (1978) for pressure in an eccentric non-rotating annular seal. The seal length-to-diameter ratio was 0.16 and the axial Reynolds number was about an order of magnitude larger than the tangential. He found that in seals with a small axial Reynolds number the pressure drop though the seal was mainly due to friction effects, but as the leakage rate increased the Bernoulli effect became stronger and high pressure gradients existed through the seal. At very high flow rates though, large head losses occurred at the entrance to the seal and the pressure distribution throughout the seal tended to be constant. The load-carrying capacity of the seal was small for the low flow rate case, increased as the flow rate increased, then peaked where the pressure gradients were high, and then decreased as the flow rate increased.

Hashimoto et al. (1988) investigated short, eccentric journal bearings to find the effect of fluid inertia on trajectory. This was basically an investigation of whether laminar flow theory would suffice in turbulent conditions. The length-to-diameter ratio of the journal was 0.5 . The averaged form of the momentum and continuity equations was used in the analysis and reaction forces were calculated for the fluctuating pressure term. Through numerical simulation the trajectory of the journals was calculated and it was demonstrated that fluid inertial effects help contribute to the stability of the system.

Kanemori and Iwatsubo (1989) considered a long annular seal with a length-to-diameter ratio of 3.0. The eccentricity of the shaft was adjustable and misalignment was not considered. They concluded that for small axial flow rates the results were similar to Fritz's theory, which did not take axial flow into account, that the reaction force in the seal was dependent on the whirl velocity. It was further concluded that the tangential reaction force was destabilizing for whirl ratios between zero and $1 / 2$, and finally that the seal is unstable at small $\mathrm{Re}_{x}$, but stable at higher Reynolds numbers. The amplitude of the tangential reaction force was minimized when the whirl ratio was $1 / 2$.

Simon and Frene (1989) studied convergent and divergent annular seals with a cryogenic working fluid. They considered the compressibility and variable viscosity of the fluid, inlet swirl and pressure drop effects. The initial assumption was that inertial effects of the fluid in the seal could be neglected. A numerical example was run with axial Reynolds numbers about 130,000 and 160,000 . This example indicated the utility of the variation of the fluid properties in the analysis. Their results were in good agreement with experimental data.

Tam et al. (1988) conducted a numerical study of eccentric annular seals without misalignment, using a model of the fluid forces based on the average fluid circumferential velocity ratio. The model was based on the assumption that the dynamic forces were rotating at the precession speed. The numerical grid was three dimensional and rotated with respect to a stationary observer at the precession rate of the rotor, i.e., the rotor was a stationary reference frame. The grid consisted of $12 \times 6 \times 16$ cells in the tangential, radial, and axial directions, and was considered invariant in time since the orbit was circular and periodic. The Prandtl mixing length turbulence model was selected because of its simplicity and because the mixing length was obviously the clearance of the seal. The eddy viscosity concept was used to simulate the effect of the turbulence levels on the mean velocities, defined in Eq. [2].

$$
\mu_{t}=\rho l_{m}^{2} \varphi
$$

The eddy viscosity $\mu_{t}$ was added to the fluid viscosity in the equations to account for the diffusive

$$
\begin{array}{ll}
y / \zeta \leq \alpha / b ; & l_{m} / \zeta=b y / \zeta, \quad b=0.435 \\
y / \zeta>\alpha / b ; & l_{m} / \zeta=\alpha, \quad \alpha=0.09
\end{array}
$$

effect of the turbulent eddies. The mixing length $l_{m}$ was defined in terms of the normal distance between the stator and rotor surfaces $\zeta$, the strain rate $\varphi$, and the minimum local distance measured from the rotor and stator surfaces, $y$.

The pressure drops ranged from 0.3 to 18.5 bars, the rotational shaft speeds from 1442 to $5085 \mathrm{rpm}$, the eccentricity ratios from 0.24 to 0.8 , and the whirl ratios from 0.3 to 1.0. Bromotriflouromethane and oil were modeled as the working fluids.

The results of the study indicated that there were significant changes in the local values of the seal dynamic forces and that large tangential separation zones existed through the seal. The existence of the recirculation zones was found to depend on the shaft and precession speeds, with increasing likelihood at lower shaft speeds and higher precession speeds (whirl ratio approaching 1.0). Recirculation zones were located along the stator wall, beginning just prior to the minimum clearance location, and rotated at the precession speed. Negative preswirl intensified the recirculation zones while preswirl in the direction of rotation weakened the secondary flow.

The effects of eccentricity and rotation speed on leakage flow rate were also studied. Leakage was most dependent on rotational speed when the precession speed was low. Increasing precession speed increased the leakage rate through the seal because of a pumping effect. 
Fluid injection from four stator positions into the clearance was also studied, and it was found that injection in the direction of the shaft rotation increased leakage while injecting against the rotation decreased leakage. Injection essentially mimicked the preswirl effects mentioned earlier, but downstream of the inlet.

In summary, previous studies showed that eccentrically aligned rotors affect annular seals in several ways. The core circulation in an eccentric seal is the same as in concentric seals (Lessen, 1987). Whirling rotors increase the leakage flow rate through seals, presumably due to a pumping effect (Chen and Jackson, 1984 and Tam, 1988). High leakage rates through the seal decrease the axial pressure gradient across the rotor because of high head losses in the entrance region (Allaire, 1978). High turbulence levels enhance the stability of whirling journals, suggesting that once whirling begins it may be damped out (Hashimoto et al., 1988). Seals are unstable at low axial Reynolds numbers (below 2000) (Allaire et al., 1978 and Kanemori \& Iwatsubo, 1989). Reaction forces are destabilizing when the whirl ratio is less than 0.5 (Kanemori \& Iwatsubo, 1989) for plain annular seals with the same surface roughness on stator and rotor. Tangential recirculation zones discovered in whirling seals during certain operating conditions were seen to diminish if there was preswirl or fluid injection in the direction of rotor rotation, and were enhanced if preswirl or injection opposed the rotor circulation direction (Tam, 1988).

\section{OBJECTIVES}

The difficulty facing many simulating the flow inside seals is knowing exactly which assumptions are valid. Measuring the velocity field inside the whirling and non-whirling seals is the only means to test the validity of assumptions used in the analysis of other researchers. For example, it is highly unlikely that the axial velocity distribution in the seal can be modeled as a plug flow as hypothesized by Lessen. Measurements are made to evaluate these assumptions and to illustrate differences that occur between eccentric whirling and non-whirling smooth annular seals. The 50\% eccentricity case was chosen as an extreme case to fully illustrate variations.

\section{EXPERIMENTAL FACILITY}

The test facility uses water at $30.5 \pm 4^{\circ} \mathrm{C}$ which is supplied from a $15 \mathrm{~m}^{3}$ storage tank by a centrifugal pump, via water filters, and a turbine meter. The flowrate through the test section is set at a constant value of $4.86 \pm 0.051 / \mathrm{s}$ which correspond to a Reynolds number of 24,000 . The test section upstream pressure is maintained at $138 \mathrm{kPa}$ to prevent cavitation and the downstream pressure is varied to control the leakage rate. The measurements are made using the seal test rig shown in Figure 2. This unit consists of a $50.8 \mathrm{~mm}$ diameter overhung shaft which is driven by a 0 to $5,300 \mathrm{rpm}$ variable speed electric motor. For the present study, the shaft rotation rate used was $3,600 \mathrm{rpm}$ which correspond to a Taylor number of 6,600. The axes of the shaft and stator housing are parallel.

The plain annular seal consists of a $35.56 \mathrm{~mm}$ long acrylic rotor mounted in a stainless steel stator. The outer diameter of the rotor is $164.1 \mathrm{~mm}$ and the inner diameter of the stator is $166.64 \mathrm{~mm}$. It was manufactured of acrylic, has a polished smooth surface, and is coated with an

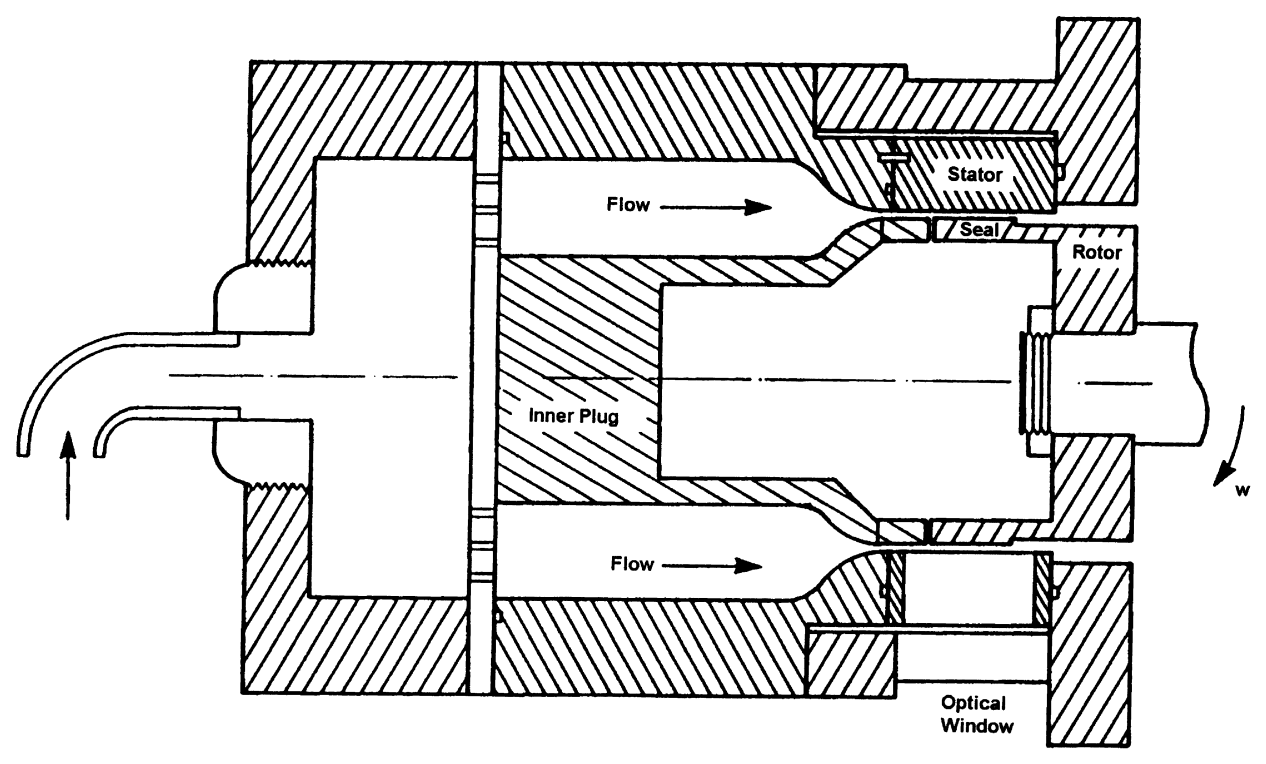

FIGURE 2 Seal test facility. 
anti-reflecting material. The stator is made of stainless steel and is mounted inside the main housing such that its position could be varied to set static eccentricities. In order to simulate a shaft that is whirling at the same speed as the shaft rotation, the seal rotors are mounted upon bushings that mount on the shaft. The bushings are machined such that their outer surfaces have eccentricities with respect to the inner shaft mounting hole of $0,10,25$ and $50 \%$ of the seal clearance. The plain annular seal is mounted on these bushings. For the present study the $50 \%$ eccentricity is used for the whirling seal. The $0 \%$ eccentric bushing is used for the non-whirling seal and the stator housing is offset to produce a stationary eccentricity of $50 \%$.

A $50.8 \mathrm{~mm}$ long laser beam access slot was cut into the stator and an optical window was placed in the trapezoidal slot which was $18.8 \mathrm{~mm}$ wide on the outside, $3.55 \mathrm{~mm}$ wide on the inside surface, and $62.23 \mathrm{~mm}$ long. The length of the window insured that inlet and exit velocity profiles could be measured. The inside and outside window surfaces were flat and parallel to $1 / 4$ of a light wavelength (approximately $125 \mathrm{~nm}$ ) to prevent any lens effects. The ratio of inside slot width and stator diameter, a measure of stator out-ofroundness, was 0.021 .

To prevent reflections from the bottom surface of the window, which would have increased the distance from the stator at which measurements could be made, the optical window was coated for maximum transmissibility for the wavelengths of the laser beams $(476.5 \mathrm{~nm}, 488 \mathrm{~nm}$, and $514.5 \mathrm{~nm}$ ). The reflectance of the window through the laser wavelengths varied almost linearly from $4 \%$ to $1 \%$. The coatings allowed measurements to $0.15 \mathrm{~mm}$ from the window surface because of the reduced glare normally seen on the surface.

The water first enters into a plenum. It then passes through a $12.7 \mathrm{~mm}$ thick perforated plate with $3.2 \mathrm{~mm}$ diameter holes which eliminates any swirl generated by the piping system, and evenly distributes the flow around the circumference of an annular plenum. The section of the annular plenum along the centerline of the test rig is necessary to provide the inner half of the contraction leading up to the seal entrance. The contraction from the plenum results in an annular flow path with a clearance of $1.524 \mathrm{~mm}$. There is a $0.508 \mathrm{~mm}$ gap between the inlet contour assembly and the rotor. The velocity distribution across the clearance at this gap was measured by Johnson and Morrison (1990). The clearance between the rotor and stator is $1.27 \mathrm{~mm}$. The larger supply nozzle clearance, $1.524 \mathrm{~mm}$, was made to accommodate eccentric seals without their inlet clearance being larger than the supply nozzle.

The seal/rotor is made of acrylic and has the following properties (Johnson, 1989): Young's modulus, $E=1.24 \times$ $10^{10} \mathrm{~Pa}$; density, $\rho=913 \mathrm{~kg} / \mathrm{m}^{3} ; \quad$ ultimate strength, $\sigma_{\text {ult }}=9.507 \times 10^{10} \mathrm{~Pa}$; coefficient of thermal expansion, $\alpha=41 \times 10^{-6} \mathrm{~m} / \mathrm{m}^{\circ} \mathrm{C}$. The seal's mechanical growth at $3,600 \mathrm{rpm}$, estimated using a hoop stress analysis of a thin cylindrical body, is $0.0386 \mathrm{~mm}$ ( $3 \%$ of the clearance), and the thermal growth is $0.006 \mathrm{~mm} /{ }^{\circ} \mathrm{C}(0.4 \%$ of the clearance for the $8^{\circ} \mathrm{C}$ test temperature window).

Seed particles were added to the water to facilitate the velocity measurements. The seed was a necessary part of the laser Doppler system, reflecting the laser light to the receiving optics. Suspended particles naturally found in the water did not reflect enough light to show up against the background noise. The seed particle selected for the experiments was Expancel 461 (Johnson, 1989). The average size of each particle is $6 \mu \mathrm{m} \pm 0.2 \mu \mathrm{m}$, and in water it has a frequency response of $44 \mathrm{Khz}$, which was sufficient to follow the turbulent fluctuations of the flow. The specific gravity of the particle was 1.29 . The particles are almost neutrally buoyant, so they behave in much the same way as water. Only two cups of the particles were needed to sufficiently seed the entire tank.

\section{Laser Doppler Velocimeter}

The layout for the laser Doppler velocimeter (LDV) was developed (Johnson, 1989) specifically for use with the seal rig. This velocimeter was configured in $30^{\circ}$ off-axis back scatter mode to enhance the signal-to-noise ratio near solid surfaces. The LDV operates on the Doppler principle: when particles pass through the intersection of two coherent light beams crossed in space, they reflect light that is Doppler shifted proportionally to their velocity. Using three different colors of light, three different frequencies are measured in three different directions.

Coherent light was produced by a $4 \mathrm{~W}$ Spectra-Physics argon-ion laser. Argon-ion laser beams contain about seven different coherent light frequencies, three of which are used to measure three different velocity components. The light from the laser was split into the individual colors using a prism. Each optical train for an individual color consisted of a polarization rotator, a beam splitter, a $40 \mathrm{MHz}$ Bragg cell, an $8.5 \mathrm{X}$ beam expander and a $150 \mathrm{~mm}$ diameter lense with a $450 \mathrm{~mm}$ focal length used to focus the beams at the measurement location. The focusing lenses were also used to collect reflected light whose light intensity was measured using a photomultiplier tube.

The output of each multiplier tube, called a Doppler burst, was analyzed using counter systems interfaced to a rotary encoding system and a computer. Electronic filters were set to assure coincidence of velocity measurements for all three velocity components to within $10 \mu \mathrm{sec}$. The computer system was used to phase average the data to obtain velocity field information at several rotor angles.

\section{EXPERIMENTAL RESULTS}

The coordinate system used to present the results was selected such that the azimuthal angle, $\theta$, was $0^{\circ}$ at the 
minimum clearance and $90^{\circ}$ on the side of the rotor where the actual pressure was a maximum. This means that physically, this location switches from one side of the rotor to the other depending on whether the rotor is statically eccentric or whirling. Figures 3 and 4 illustrate this. The statically eccentric seal, presented in Figure 3, generates a pressure distribution in the same way as a journal bearing. As the fluid is dragged into the converging channel on the left side of the seal, the pressure increases. As it emerges from the minimum clearance into the diverging section, the pressure decreases. Hence the pressure side is on the left. For the whirling seal, the fluid motion is not caused primarily by the viscous drag, but by the dynamic action of the rotor created by the seal sweeping through the fluid. This action generates a high pressure region on the "leading edge" of the rotor as it pushes the fluid around the seal as opposed to the dragging action present in the non whirling seal. The rotor shown in Figure 4 is precessing in a clockwise direction. Hence, the minimum clearance is moving clockwise. The squeezing of the fluid into a converging channel by the motion of the rotor, causes the pressure to increase on the right hand side. The fluid on the left hand side is in the wake of the rotor and has a low pressure. Another way to see the whirling seal effect is to examine how they are modeled in a computational fluid dynamics program. In this case, the time dependent flow field (Tam, 1988; Robic, 1999) is converted into a steady state condition by placing the coordinate frame on the rotor. Then the stator rotates in a counter clockwise direction. Hence, the pressure side is on the right hand side.

The mean axial velocity radial distribution for rotor angles of $0^{\circ}, 90^{\circ}, 180^{\circ}$, and $270^{\circ}$ are presented in Figures 5-7 along with the average value across the clearance and how it varies along the seal length. The radial direction has been nondimensionalized by the nominal seal clearance

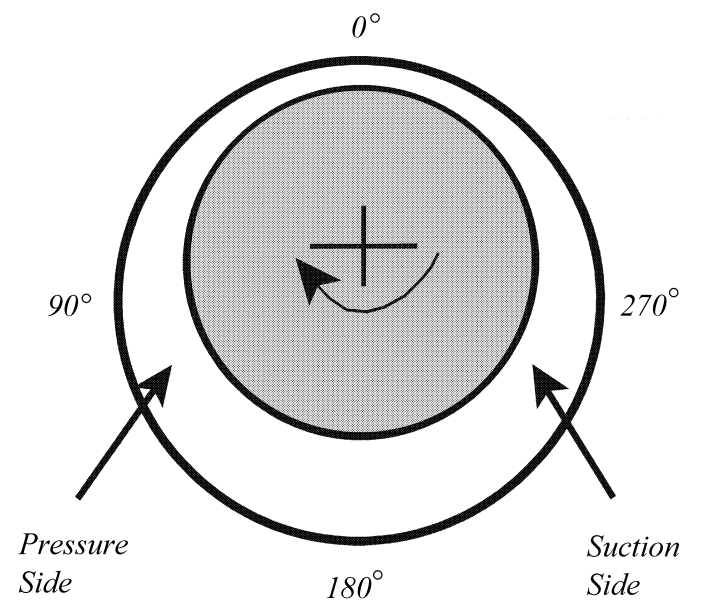

FIGURE 3 For the statically eccentric seal the rotor viscous stresses drag the fluid in the direction of shaft rotation generating pressure on the left and suction on the right.

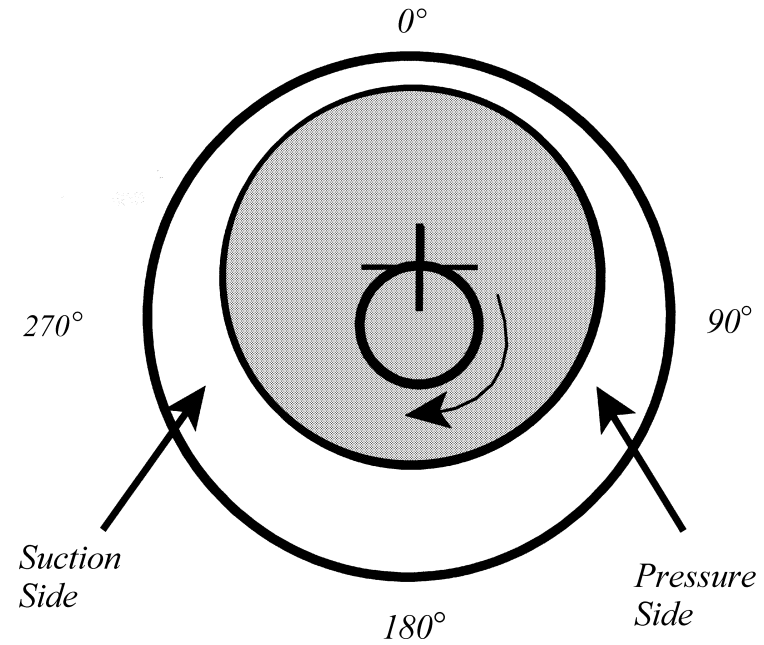

FIGURE 4 For the eccentric seal whirling at a whirl ratio of one the rotor pushes the fluid around the seal generating pressure on the front face of the seal (right side) and suction (left side) in the wake of the rotor.
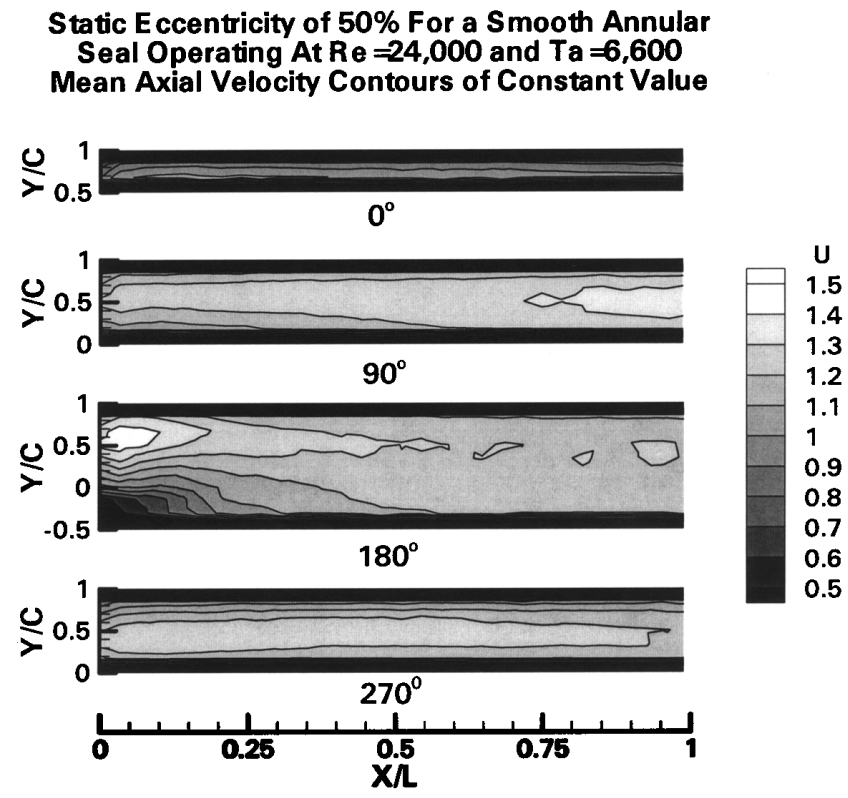

FIGURE 5 Mean axial velocity contours for a statically eccentric smooth annular seal.

and the velocity has been nondimensionalized by the bulk averaged leakage velocity. At the minimum clearance there is little difference between the two conditions with the axial velocity maximizing at $1.1 U_{\text {ave }}$ in the center of the clearance along the entire seal length with an average value near $0.6 U_{\text {ave }}$ along the seal length. On the pressure side $\left(90^{\circ}\right)$ of the seal, the high pressure resists the entrance of the flow into the seal with lower values near the rotor surface. However, the effect is significantly larger for the whirling seal where the "bulk averaged" axial velocity 


\section{Whirling Eccentricity of $50 \%$ For a Smooth Annular Seal Operating at a Whirl Ratio of One, $R e=24,000$ and $\mathrm{Ta}=6,600$ Mean Axial Velocity Contours of Constant Value}
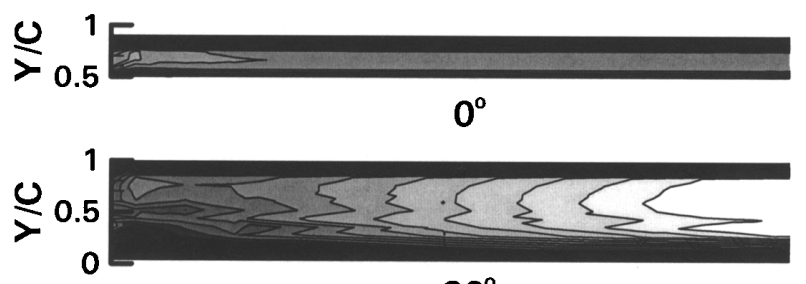

$90^{\circ}$

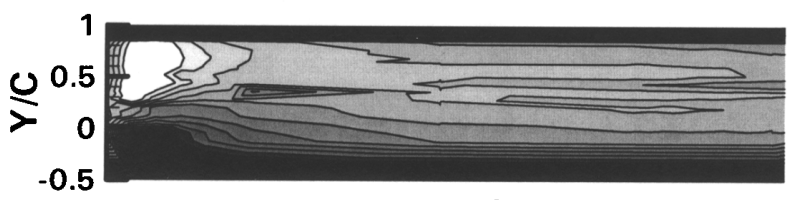

$180^{\circ}$ $\mathbf{U}$
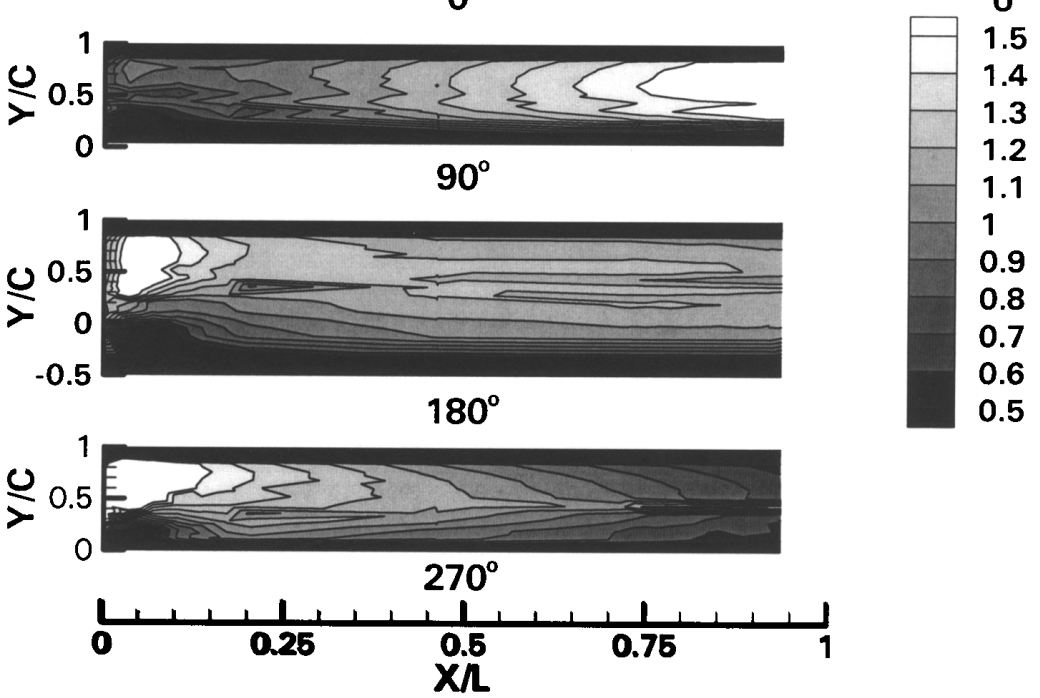

FIGURE 6 Mean axial velocity contours for a whirling smooth annular seal.

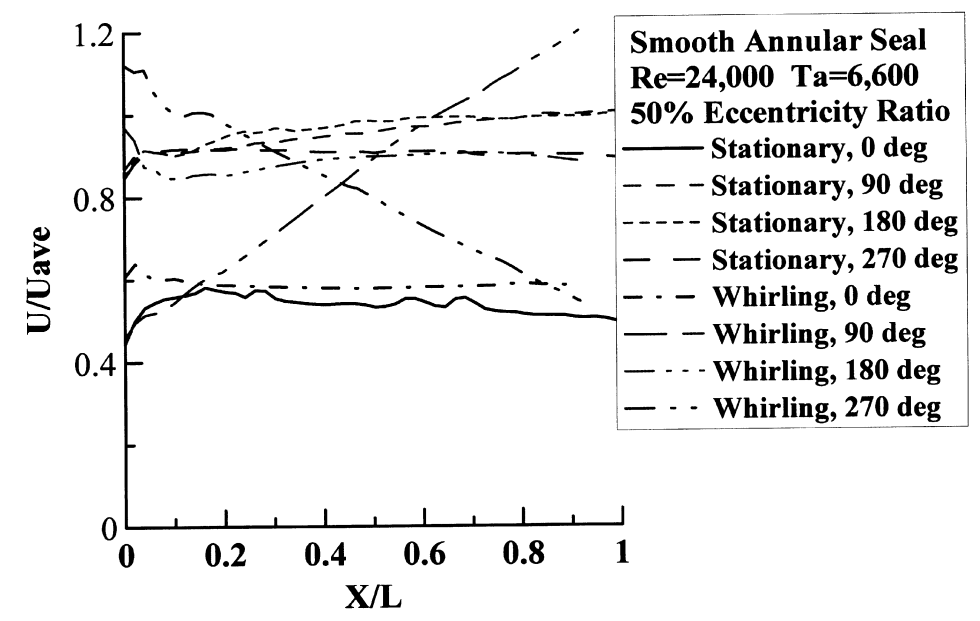

FIGURE 7 "Bulk averaged" mean axial velocity distribution along the seal length.

is only $0.5 U_{\text {ave }}$ compared to $0.9 U_{\text {ave }}$ for the statically eccentric seal. Interestingly enough, for both cases, the axial velocity increases on the pressure side as the flow progresses down the seal with "bulk averaged" values of $1.2 U_{\text {ave }}$ and $1.0 U_{\text {ave }}$ for the whirling and stationary seals respectively at the seal exit. On the suction side of the seal $\left(\theta=270^{\circ}\right)$ exactly the opposite occurs with the axial velocity entering the seal at its highest value and decreasing as the flow progresses downstream. In this case, the "bulk averaged" inlet velocity is $1.1 U_{\text {ave }}$ for the whirling seal and $0.9 U_{\text {ave }}$ for the statically eccentric seal. At the exit, the statically eccentric seal "bulk averaged" axial velocity has shown only a small decrease in value while the "bulk averaged" axial velocity has decreased to $0.5 U_{\text {ave }}$ for the whirling seal. At the maximum clearance position $\left(\theta=180^{\circ}\right)$, the axial velocity contours are very similar for 
both the whirling and non-whirling seal with a large variation in axial velocity across the inlet plane (from $0.5 U_{\text {ave }}$ to $1.5 U_{\text {ave }}$ ) which becomes more uniform as the flow progresses downstream. The axial velocity maximizes near the stator and is a minimum near the rotor. The "bulk averaged" velocity does not vary significantly with axial position at the maximum clearance remaining around $0.9 U_{\text {ave }}$ for the whirling seal and $0.95 U_{\text {ave }}$ for the nonwhirling seal.

Winslow (1994) has shown that for the whirling seal, the change in highest axial velocity location from the suction side of the seal at the entrance to pressure side of the seal at the exit is accompanied by a switch in the low pressure at the inlet on the suction side to the low pressure being present on the pressure side of the seal at the seal exit.

The azimuthal axial velocity (nondimensionalized by the bulk averaged leakage velocity) contours for the statically eccentric seal (Figure 8) and the whirling seal (Figure 9) demonstrate how the whirling seal significantly increases the azimuthal velocity at all locations inside the seal. The whirling seal even produces an effective pre-swirl upstream of the seal. For the statically eccentric annular seal, the azimuthal velocity is large near the rotor surface with almost no azimuthal velocity present in the incoming flow. As the flow progresses downstream, the azimuthal velocity begins to increase across the clearance as the viscous drag effect continues to increase its influence across the clearance as the fluid residence time increases, i.e., in the downstream direction. The whirling seal does not exhibit this ever increasing effect as markedly as the statically eccentric seal as shown by the contour lines remaining almost parallel to the rotor wall showing only a slight increase in the downstream direction except of on the suction side of the seal $\left(\theta=270^{\circ}\right)$ past $X / L=0.5$. In this region the axial velocity has slowed considerably and the residence time of the fluid in this region is increased significantly due to the lower axial speed. This allows the viscous drag of the rotor to exert more influence upon the fluid and increase its azimuthal velocity.

The "bulk averaged" azimuthal velocity values along the length of the seal are shown in Figure 10. These data show how the whirling seal greatly increases the inlet pre-swirl compared to the statically eccentric seal. The statically eccentric seal exhibits inlet "bulk averaged" azimuthal values from $0.5 U_{\text {ave }}$ to $0.8 U_{\text {ave }}$ while the whirling seal values range from $0.8 U_{\text {ave }}$ to $1.3 U_{\text {ave. }}$. Please note that the closest measured values to the rotor were $0.16 c$ above the rotor. In this region, the azimuthal velocity was assumed to vary linearly. This results in an overestimation of the "bulk averaged" azimuthal velocity. These values can, however, be used for comparison purposes to see overall effects of whirling versus non-whirling seals. The rate of increase of the "bulk averaged" azimuthal velocity is relatively constant for all azimuthal locations for the statically eccentric seal with an increase of $0.4 U_{\text {ave }}$. The whirling seal shows less increase, $0.2 U_{\text {ave }}$ to $0.4 U_{\text {ave }}$, but maintains

\section{Static Eccentricity of $50 \%$ For a Smooth Annular Seal Operating At $R e=24,000$ and $\mathrm{Ta}=6,600$ Mean Azimuthal Velocity Contours of Constant Value}
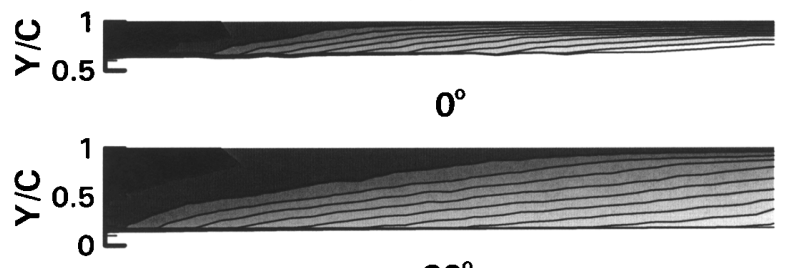

$90^{\circ}$
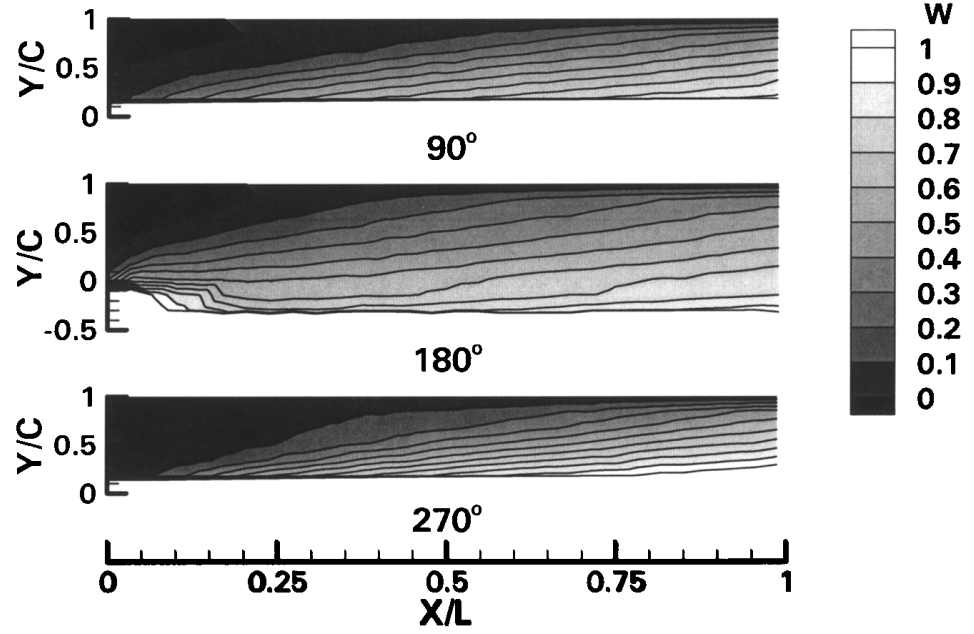

FIGURE 8 Mean azimuthal velocity contours for a statically eccentric smooth annular seal. 


\section{Whirling Eccentricity of $50 \%$ For a Smooth Annular Seal \\ Operating at a Whirl Ratio of One, $\operatorname{Re}=24,000$ and $\mathrm{Ta}=6,600$ Mean Axial Velocity Contours of Constant Value}
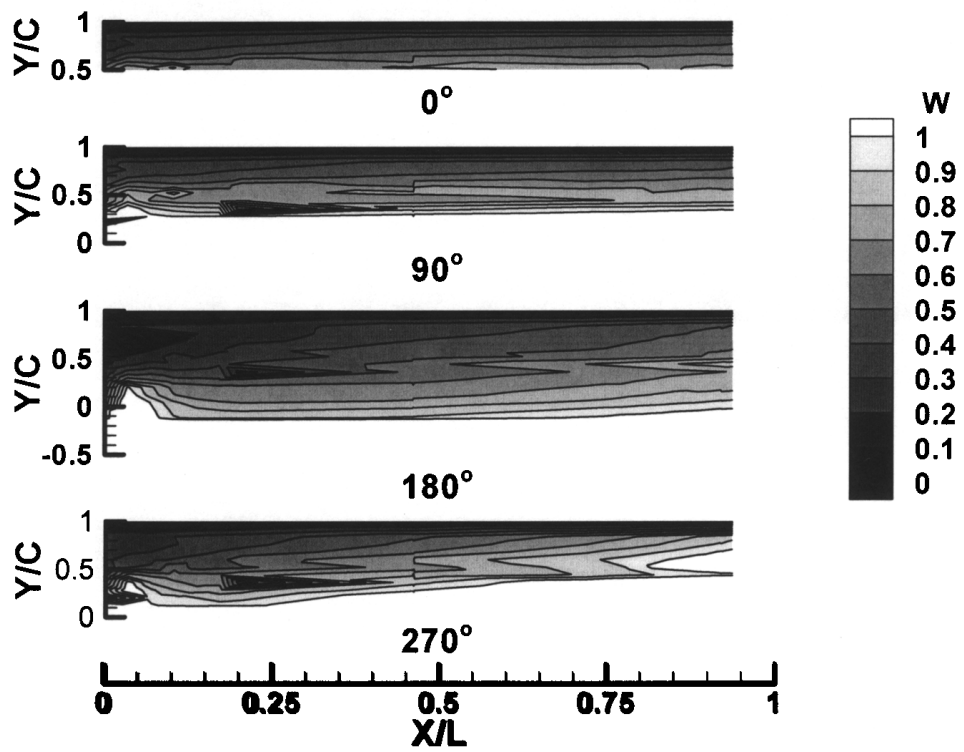

FIGURE 9 Mean azimuthal velocity contours for a whirling smooth annular seal.

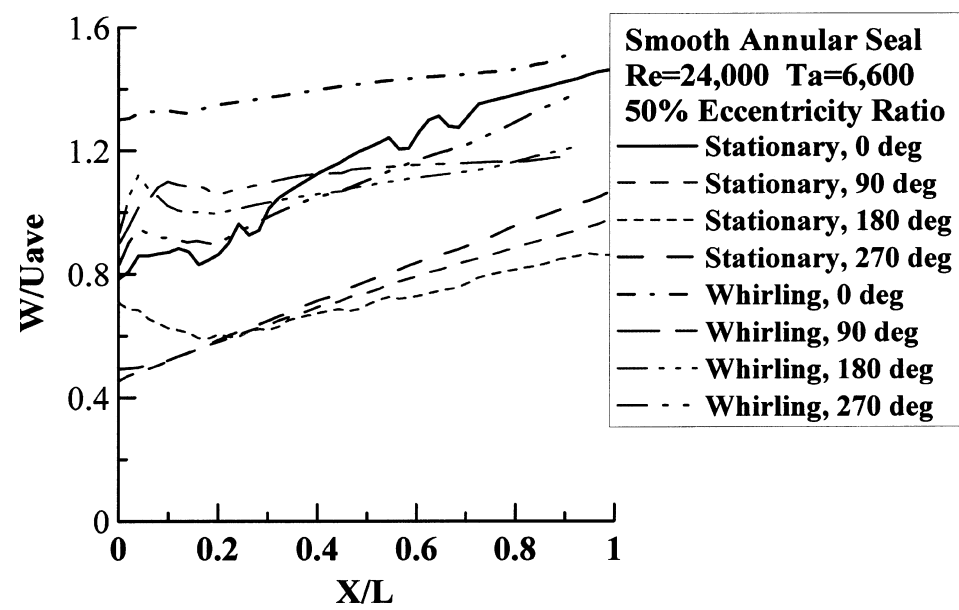

FIGURE 10 "Bulk averaged" azimuthal mean velocity distribution along the seal length.

values that are larger than the non-whirling seal at comparable azimuthal locations.

The turbulence kinetic energy contours nondimensionalized by $U_{\text {ave }}^{2}$ are shown in Figures 11 and 12. For both the whirling and non-whirling seal, the turbulence in the minimum clearance region maximizes at $0.12 U_{\text {ave }}^{2}$. However, the turbulence level steadily increases from essentially zero to this maximum value at the seal exit for the nonwhirling seal. This turbulence is generated by the large velocity gradient generated by the seal rotor which is providing sufficient energy to maintain a net production of turbulence along the seal length. The whirling seal is generating turbulence upstream of the seal due to its mixing action which is convected into the seal. Some of this turbulence decays as the flow progresses in the downstream direction until $X / L=0.2$. The azimuthal region of maximum turbulence level is between $200^{\circ}$ and $270^{\circ}$ with an axial distribution as shown in Figure 12. At this point the action of the rotor inside the seal is imparting sufficient energy to the turbulence production mechanism such that the turbulence level increases to the exit of the seal. The turbulence levels in the non-whirling and whirling seal are 


\section{Static Eccentricity of $50 \%$ For a Smooth Annular \\ Seal Operating At $\mathrm{Re}=24,000$ and $\mathrm{Ta}=6,600$ \\ Contours of Constant Turbulence Kinetic Energy Value}
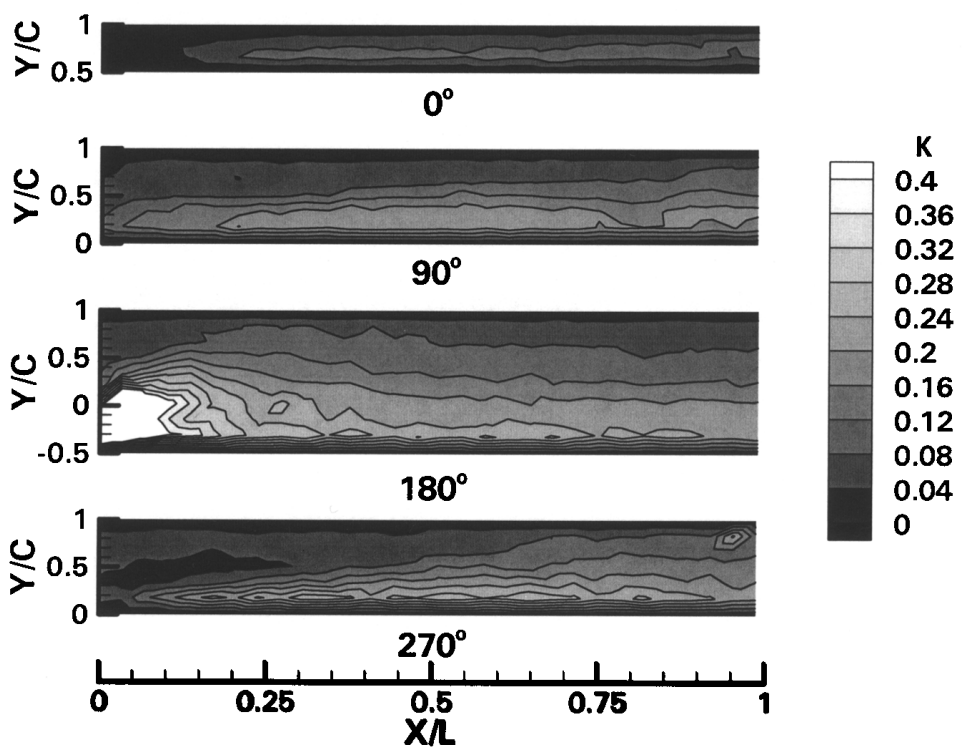

FIGURE 11 Turbulence kinetic energy levels for a statically eccentric smooth seal.

Whirling Eccentricity of $50 \%$ For a Smooth Annular Seal Operating at a Whirl $R$ atio of One, $R e=24,000$ and $\mathrm{Ta}=6,600$

Contours of Constant Turbulence Kinetic Energy Value
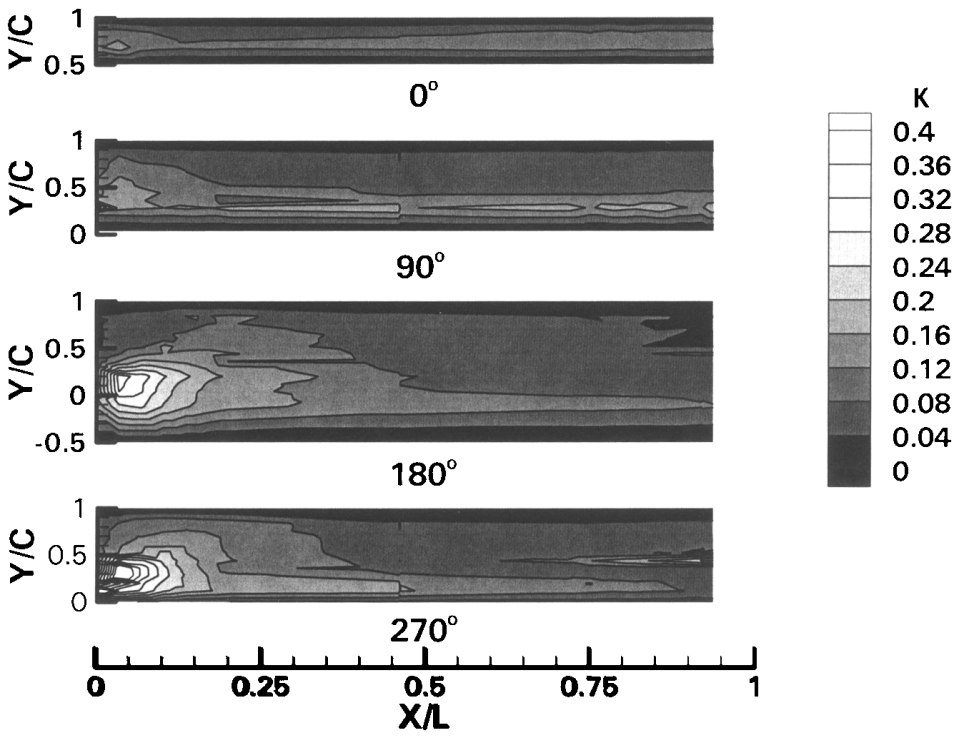

FIGURE 12 Turbulence kinetic energy levels for a whirling smooth annular seal.

generated and/or maintained by the radial gradient of the mean velocity in the last half of the seal. The non-whirling seal's turbulence levels are larger near the seal exit than for the whirling seal due to the larger velocity gradients present since the bulk averaged azimuthal velocities in the nonwhirling seal is only $65 \%$ of the whirling seal values.

For the statically eccentric seal, a similar sequence of events is present at all azimuthal locations with the 


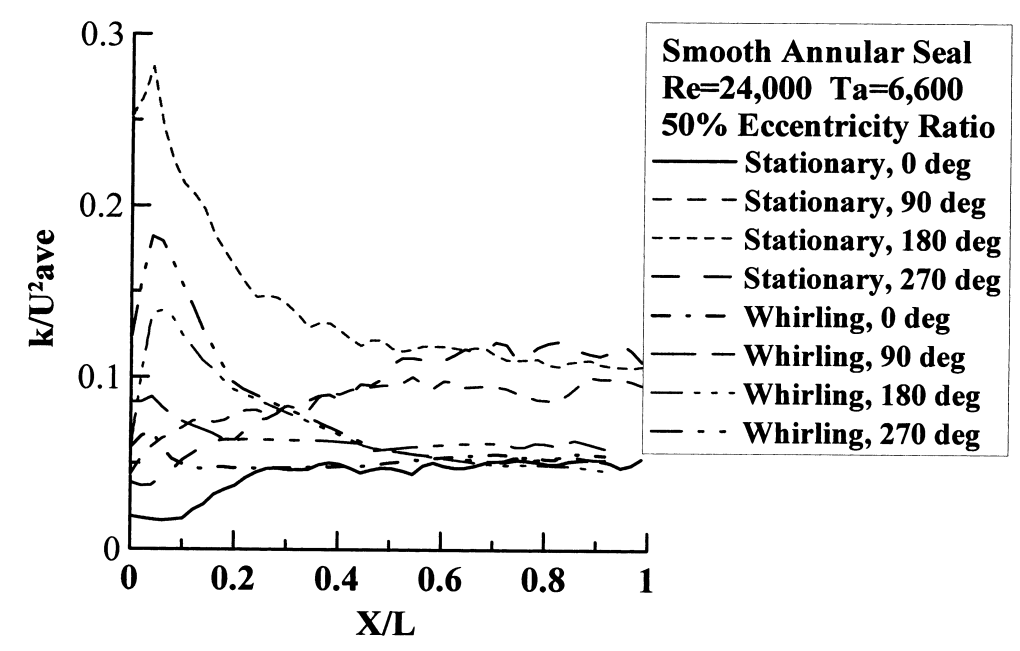

FIGURE 13 "Bulk averaged" turbulence kinetic energy distribution along the seal length.

maximum level of turbulence kinetic energy increasing to $0.24 U_{\text {ave }}^{2}$. There is one difference, and that is at the location of maximum clearance. Near the rotor surface at the seal inlet there is a very large level $\left(0.4 U_{\text {ave }}^{2}\right)$ which corresponds to the location where the axial velocity increases rapidly from $0.5 U_{\text {ave }}$ to $1.5 U_{\text {ave }}$ across the seal clearance. This axial velocity variation is due to the seal inlet design condition and the locally very large velocity gradients produce extremely large levels of turbulence. For the whirling seal, the flow field development at the larger clearances is modified with the turbulence generated by the entrance effects into the seal (due to both entering the seal and the mixing produced by the whirling of the seal) result in the maximum amount of turbulence being present from $X / L=0$ to 0.2 with values maximizing at $0.4 U_{\text {ave }}^{2}$. These values decrease rapidly as the flow progresses downstream and remain relatively constant from $X / L=0.4$ to the seal exit. This shows how the whirling seal turbulence production mechanism is dominated by the inlet mixing effects with the greater increase in azimuthal velocity, which effectively decreases the turbulence production downstream since the radial velocity gradients have been significantly reduced. Figure 13 presents the "bulk averaged" values of the turbulence kinetic energy. These data show how the statically eccentric seal produces more turbulence in the downstream $2 / 3$ length of the seal than the whirling seal.

\section{CONCLUSIONS}

These data show that the flow field inside whirling and non-whirling seals can be dramatically different. The statically eccentric seal is dominated by viscous drag which is responsible for generating the azimuthal velocity inside the seal and the turbulence. Conversely, the whirling seal produces the azimuthal velocity component by pushing the fluid by the rotor (dynamic forces) which result in larger azimuthal velocity values, decreased radial velocity gradients, and decreased turbulence production inside the seal. The whirling seal generates the majority of its turbulence in the entrance region while the non-whirling seal does this in the last $2 / 3$ of the seal length. The biggest difference is that the region of high pressure switches from one side of the seal to the other. This means that the forces generated by the seal will be in opposite directions. It also implies that at whirl ratios between 0 and 1 the magnitude of the pressure and suction pressures must decrease in value from the statically eccentric values, reach a minimum, and then increase again only on the opposite sides of the seal. This can explain why the value of the whirl ratio can determine if a seal will be stabilizing or destabilizing. The axial velocity distributions in the first third of the seal cannot be represented by plug flow due to the entrance effects. However, the last two thirds of the seal plug flow does fairly well represent the axial velocity distributions with the magnitude changing as the flow progresses through the seal. The azimuthal velocity cannot be represented by plug flow anywhere in the seal. There is always a significant radial variation in the azimuthal velocity.

\section{NOMENCLATURE}

$c$ nominal clearance between rotor and stator $=$ $1.27 \mathrm{~mm}$

D rotor diameter $=164.1 \mathrm{~mm}$

e rotor eccentricity ratio, eccentricity $/ c$ 
$k \quad$ turbulence kinetic energy $/ U_{\text {ave }}^{2}$

$L \quad$ rotor length $=35.56 \mathrm{~mm}$

$P \quad$ pressure

$Q$ volumetric flow rate of fluid through the seal= $4.861 / \mathrm{s}$

Re axial Reynolds number $=2 \rho U_{\text {ave }} c / \mu$

$\operatorname{Re}_{\theta} \quad$ azimuthal Reynolds number $=2 \rho W_{\text {ave }} c / \mu$

$\mathrm{Ta}$ Taylor number $=\left(\rho W_{s h} c / \mu\right)(2 c / D)^{1 / 2}$

$U$ mean axial velocity $/ U_{\text {ave }}$

$U_{\text {ave }}$ bulk averaged leakage velocity, $Q / \pi D c=7.49 \mathrm{~m} / \mathrm{s}$

$W$ mean azimuthal (tangential) velocity $/ U_{\text {ave }}$

$W_{s h}$ rotor surface velocity $=30.9 \mathrm{~m} / \mathrm{s}=4.125 U_{\text {ave }}$ @) $3600 \mathrm{rpm}$

$X \quad$ axial distance downstream of the seal entrance

$Y$ radial distance above centered rotor surface

$\theta \quad$ azimuthal angle (see Figs. 3 and 4)

$\mu \quad$ absolute viscosity, $7.84 \times 10^{-4} \mathrm{~kg} / \mathrm{m}-\mathrm{s}$

$v \quad$ kinematic viscosity $=\mu / \rho$

$\rho \quad$ density, $999 \mathrm{~kg} / \mathrm{m}^{3}$

\section{REFERENCES}

Allaire, P. E., Lee, C. C. and Gunter, E. J. (1978) Dynamics of Short Eccentric Plain Seals with High Axial Reynolds Numbers, Journal of Spacecraft and Rockets, 15, $341-347$.

Chen, W. C. and Jackson, E. D. (1984) Eccentricity and Misalignment Effects on the Performance of High-Pressure Annular Seals, ASLE Transactions, 28, 104-110.

Hashimoto, H., Wada, S. and Sumitomo, M. (1988) Effects of Fluid Inertia Forces on the Dynamic Behavior of Short Journal
Bearings in Superlaminar Flow Regimes, Journal of Tribology, 110 $539-547$

Hirs, G. G. (1973) Bulk-Flow Lubricant Theory for Turbulence in Lubricant Films, ASME Journal of Lubricant Technology, 95, $137-146$

Johnson, M. C. (1989) Development of a 3-D Laser Doppler Anemometry System: With Measurements in Annular and Labyrinth Seals, Ph.D. Dissertation, Texas A \& M University, College Station, Texas, 77843.

Kanemori, Y. and Iwatsubo, T. (1989) Experimental Study of Dynamical Characteristics of a Long Annular Seal, JSME International Journal Series II, 32, 218- 224.

Lessen, M. (1987) Turbulent Flow in Shaft Seals and Bearings, STLE Tribology Transactions, 31, 390-396.

Morrison, G. L., Johnson, M. C. and DeOtte, R. E. (1990) Experimental Investigation of an Eccentric Labyrinth Seal Velocity Field Using 3D Laser Doppler Anemometer, Journal of Fluid Machinery Components, 101, $61-71$.

Robic, B. F. (1999) Experimental and Numerical Analysis of the Effect of Swirl on the Pressure Field in Whirling Annular and Labyrinth Seals, Ph.D. Dissertation, Texas A \& M University, College Station, Texas, 77843.

Schlichting, H. (1979) Boundary-Layer Theory, McGraw-Hill Classic Textbook Reissue, New York.

Simon, F. and Frene, J. (1989) Static and Dynamic Characteristics of Turbulent Annular Eccentric Seals: Effect of Convergent-Tapered Geometry and Variable Fluid Properties, ASME Journal of Tribology, 111, 378-385.

Stuart, J. T. (1958) On the Nonlinear Mechanics of Hydro-Dynamic Stability, Journal of Fluid Mechanics, 4, 1-21.

Thames, H. D. (1992) Mean Flow and Turbulence Characteristics in Whirling Annular Seals, M.S. Thesis, Texas A \& M University, College Station, Texas, 77843.

Tam, L. T., Przekwas, A. J., Muszynska, A., Hendriks, R. C., Braun, M. J. and Mullen, R. L. (1988) Numerical and Analytical Study of Fluid Dynamic Forces in Seals and Bearings, Journal of Vibration, Acoustics, Stress, and Reliability in Design, 110, 315-325.

Winslow, R. W. (1994) Dynamic Pressure and Shear Stress Measurements on the Stator Wall of Whirling Annular Seals, M.S. Thesis, Texas A \& M University, College Station, Texas, 77843. 

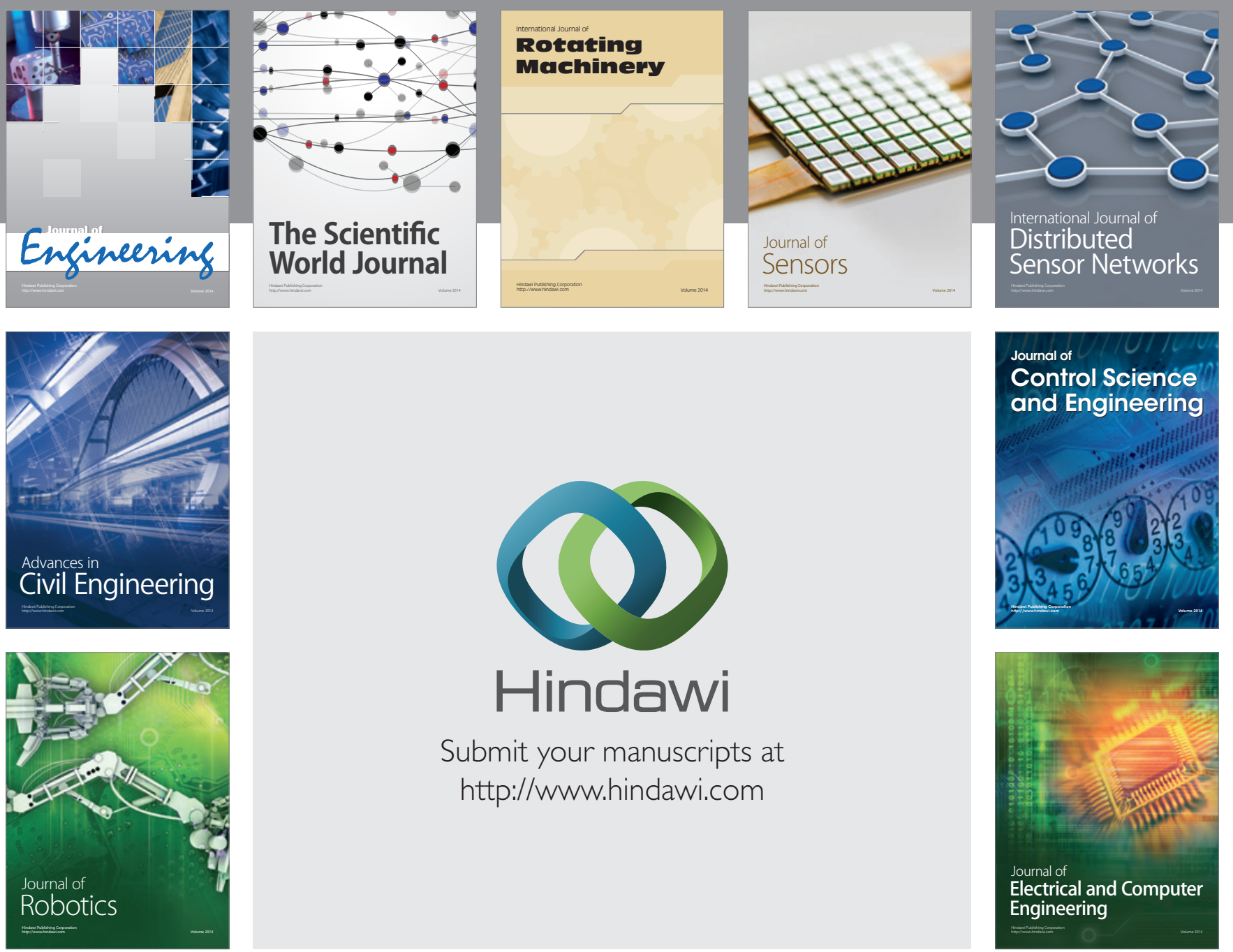

Submit your manuscripts at

http://www.hindawi.com
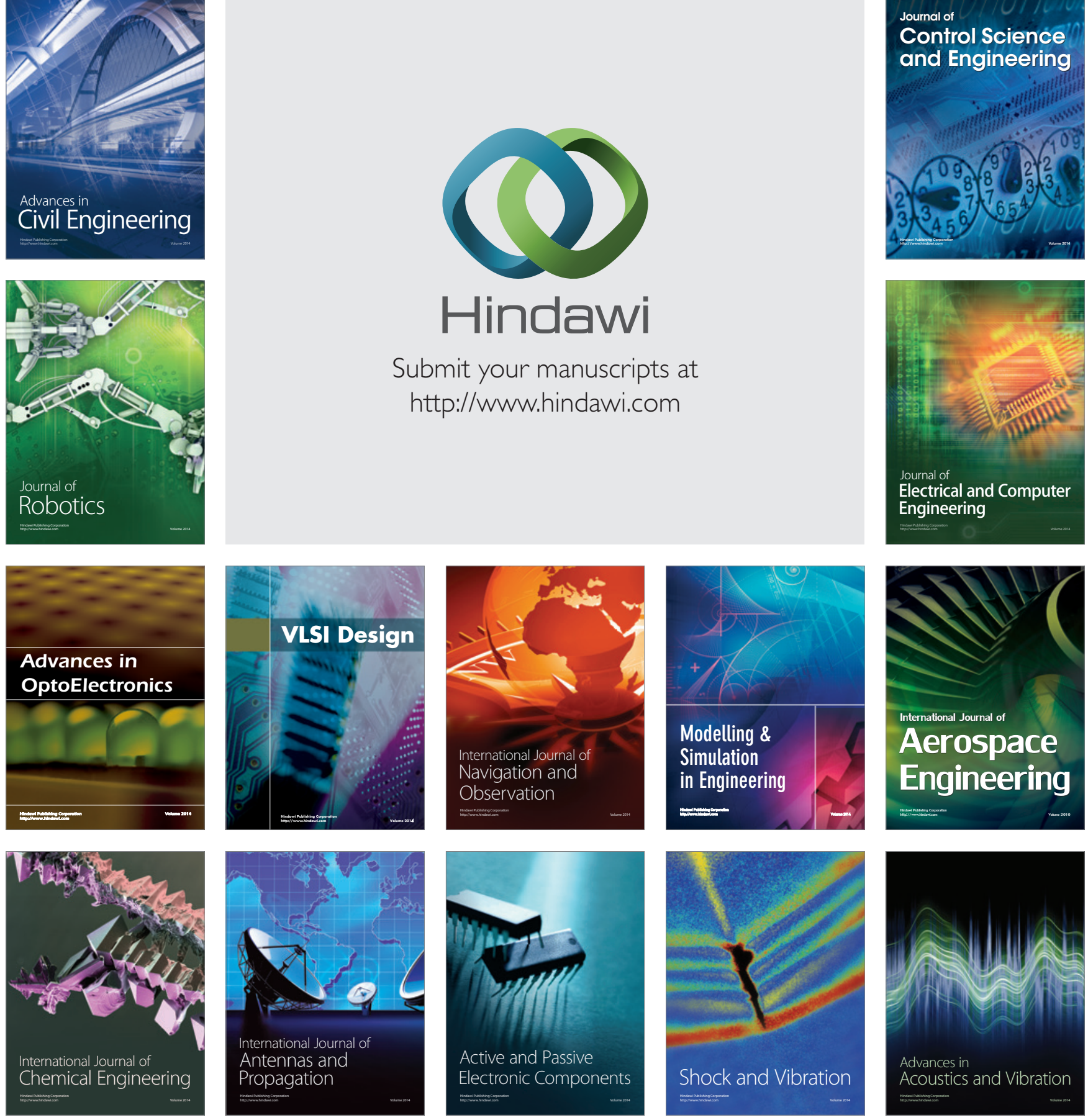